\title{
Sensitivity Studies for the Exercise I-1 of the OECD/UAM Benchmark
}

\author{
E. Canuti, ${ }^{1}$ A. Petruzzi, ${ }^{1}$ F. D'Auria, ${ }^{1}$ and T. Kozlowski ${ }^{2}$ \\ ${ }^{1}$ San Piero a Grado Nuclear Research Group (GRNSPG), University of Pisa, Via Diotisalvi 2, 56122 Pisa, Italy \\ ${ }^{2}$ Department of Nuclear, Plasma, and Radiological Engineering, University of Illinois, 216 Talbot Laboratory, MC-234, \\ 104 South Wright Street, Urbana, IL 61801, USA \\ Correspondence should be addressed to E. Canuti, erika.canuti@gmail.com
}

Received 24 August 2012; Revised 30 October 2012; Accepted 31 October 2012

Academic Editor: Oscar Cabellos

Copyright () 2012 E. Canuti et al. This is an open access article distributed under the Creative Commons Attribution License, which permits unrestricted use, distribution, and reproduction in any medium, provided the original work is properly cited.

OECD/NEA has initiated an international Uncertainty Analysis in Modeling (UAM) benchmark focused on uncertainties in modeling of Light Water Reactor (LWR). The first step of uncertainty propagation is to perform sensitivity to the input data affected by the numerical errors and physical models. The objective of the present paper is to study the effect of the numerical discretization error and the manufacturing tolerances on fuel pin lattice integral parameters (multiplication factor and macroscopic crosssections) through sensitivity calculations. The two-dimensional deterministic codes NEWT and HELIOS were selected for this work. The NEWT code was used for analysis of the TMI-1, PB-2, and Kozloduy-6 test cases; the TMI-1 test case was investigated using the HELIOS code. The work has been performed within the framework of UAM Exercise I-1 "Cell Physics."

\section{Introduction}

OECD/NEA has initiated an international Uncertainty Analysis in Modeling (UAM) benchmark focused on propagation of uncertainties in the entire modeling chain of Light Water Reactor (LWR) in steady-state and transient conditions. The final objective is to benchmark uncertainty and sensitivity analysis methods in coupled multiphysics and multiscale LWR calculations.

The present paper is concerned only with cell and lattice physics. In reactor analysis, the lattice physics calculations are used to generate nodal (lattice-averaged) parameters, used for the full-core simulation. Similarly to other numerical simulations, the lattice-averaged parameters are affected by uncertainties. In lattice physics, these uncertainties can be divided into 3 types:

(i) Multigroup cross sections uncertainties,

(ii) Uncertainties associated with methods and modeling approximations used in lattice physics codes, and

(iii) Fuel/assembly manufacturing tolerances.
The objective of the present paper is to study the effect of the last two uncertainty sources, within the framework of UAM Exercise I-1 "Cell Physics." This exercise is focused on derivation of the multigroup microscopic cross-section libraries. Even if the intention for Exercise I-1 is to propagate the uncertainties in evaluated Nuclear Data LibrariesNDL-(microscopic point-wise cross sections) into multigroup microscopic cross-sections, here the NDL data have been used directly to perform lattice physics calculations (fuel pin lattices) in order to evaluate neutronics-related parameters.

For uncertainty propagation, the first step is to perform sensitivity to the input data affected by the errors or uncertainties. In this paper, the effect of numerical discretization errors and manufacturing tolerances on fuel pin lattice integral parameters (multiplication factor and cross-sections) has been analyzed through sensitivity calculations.

The two-dimensional deterministic codes NEWT and HELIOS were selected for this work. The NEWT code was used for analysis of the TMI-1, PB-2, and Kozloduy- 6 test cases. Then, the TMI- 1 test case was investigated using the 
HELIOS code. Finally, a comparison has been made between the two lattice codes.

\section{Codes Description}

Two deterministic lattice codes have been used to perform the uncertainties studies: NEWT and HELIOS.

NEWT (New ESC-based Weighting Transport code) is a two-dimensional (2D) discrete-ordinates transport code developed at Oak Ridge National Laboratory [1]. It is based on the Extended Step Characteristic (ESC) approach for spatial discretization on an arbitrary mesh structure. This discretization scheme makes NEWT an extremely powerful and versatile tool for deterministic calculations in real-world nonorthogonal domains. The NEWT computer code has been developed to run within the SCALE package. Thus, NEWT uses AMPX-formatted cross-sections processed by other SCALE modules.

HELIOS is a generalized-geometry 2D lattice physics code developed by Studsvik-Scandpower [2]. HELIOS uses Current-Coupled Collision Probability (CCCP) method for its transport solution. The system to be calculated consists of heterogeneous space elements (e.g., pin-cell) that are coupled with each other and with the boundaries by interface currents, while the properties of each space element (i.e., its responses to sources and in-currents) are obtained from Collision Probability method (CP).

\section{Model and Reference Simulation Parameters}

The two-dimensional fuel pin-cell test problems representative of BWR PB-2, PWR TMI-1, and Kozloduy-6 VVER1000 have been analyzed. The UAM specifications were used to define these three test problems, the details are shown in Figures 1, 2 and 3 for BWR, PWR, and VVER-1000 pin-cell, respectively [3].

In addition to the cell geometry, material compositions, and material temperatures, each code requires code-specific numerical parameters. Table 1 shows the numerical parameters used for the reference simulation of the three test cases.

\section{Numerical Error and Sensitivity Calculation}

The focus of the paper is on the numerical discretization error and the manufacturing tolerance sensitivities. The specific description of each type of calculation is described in the section below.

4.1. Numerical Discretization Error. The numerical discretization error was quantified for $k_{\text {inf }}$ in the NEWT code. It was quantified for the two major numerical approximations used by NEWT code.

(1) The spatial discretization of the cell grid: users can define a computational grid in which the NEWT ESC solution algorithm is applied. Convergence studies have been performed on $k_{\text {inf }}$ value for the grid dimension from $2 \times 2$ (base model) up to $40 \times 40$ per cell.
TABLE 1: Reference simulation parameters.

\begin{tabular}{lcc}
\hline Parameter & NEWT & HELIOS \\
\hline Cross-sections library & $\begin{array}{c}\text { ENDF/B- } \\
\text { VII.0 }\end{array}$ & $\begin{array}{c}\text { HELIOS } \\
\text { master library }\end{array}$ \\
$\begin{array}{l}\text { Number of energy group } \\
\text { Grid structure }\end{array}$ & 238 & 190 \\
Number of sides per cylinder & 12 & $2 \times 2$ \\
Number of fuel pin azimuthal regions & - & - \\
Convergence Criteria & & 4 \\
$\quad \begin{array}{l}\text { Inner iterations } \\
\text { Outer iterations } \\
\quad k_{\text {inf }} \text { value }\end{array}$ & $10^{-4}$ & $10^{-4}$ \\
\hline
\end{tabular}

(2) The approximation of a circle with an equilateral polygon with a certain number of sides. The default number is 12. The influence of this approximation on the $k_{\text {inf }}$ value has been evaluated for the number of sides from 8 to 28 .

The study has been carried out for the three test cases in HZP condition only.

4.2. Manufacturing Tolerance Sensitivities. Sensitivity of lattice-averaged parameters to manufacturing tolerances has been studied using data provided by the UAM specifications for Phase I. For TMI-1 and PB-2 test cases, the manufacturing uncertainties are shown in Table 2. Normal distribution was assumed for each parameter Probability Density Function (PDF).

For VVER-1000 test case, the manufacturing tolerances are shown in Table 3. Uniform distribution was assumed for each parameter PDF.

It should be noted that these uncertainties were specified for the fuel assembly, but in this work they have been applied for the single pin-cell. The sensitivities have been performed by changing the listed parameters affected by manufacturing tolerances. For TMI-1 and PB-2 cases all the listed parameters have been increased by the quantity indicated in Table 2. For VVER-1000 case sensitivities have been performed using both the lower and the upper limits of the parameters listed in Table 3 (note that some limits correspond to the reference value). The sensitivities were calculated for the $k_{\text {inf }}$ and the macroscopic 2 group crosssections (absorption, fission, and scattering), in HZP and in HFP conditions.

It should be noted that in this work the term "sensitivity" has not been used in the usual way but as reactivity differences $\left(\rho_{2}-\rho_{1}\right)$ due to a parameter variation.

\section{Results}

5.1. Criticality Results. The reference (base case) results for the three test models are presented in this section. The lattice 


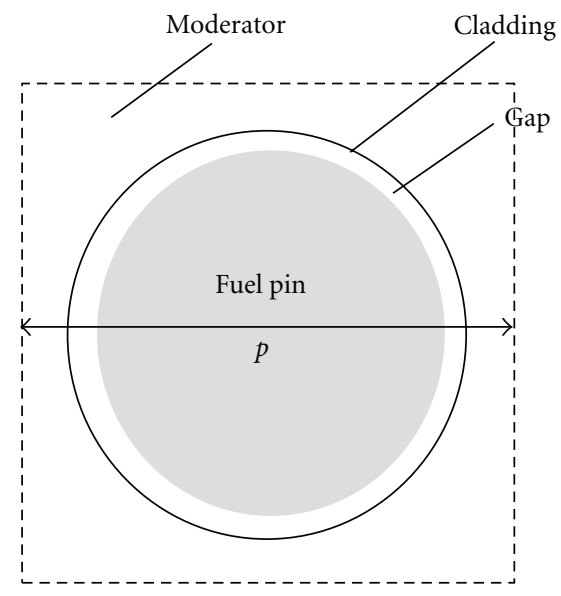

$p$-pitch of the unit cell

\begin{tabular}{lc}
\hline Parameter & Value \\
\hline Unit cell pitch, $[\mathrm{mm}]$ & 18.75
\end{tabular}

Fuel pellet diameter, $[\mathrm{mm}] \quad 12.1158$

Fuel pellet material $\quad \mathrm{UO}_{2}$

Fuel density, $\left[\mathrm{g} / \mathrm{cm}^{3}\right] \quad 10.42$

Fuel enrichment, [w/o] 2.93

Cladding outside diameter, [mm] 14.3002

Cladding thickness, $[\mathrm{mm}] \quad 0.9398$

Cladding material Zircaloy-2

Cladding density, $\left[\mathrm{g} / \mathrm{cm}^{3}\right]$

Gap material $\mathrm{He}$

Moderator material $\quad \mathrm{H}_{2} \mathrm{O}$

\begin{tabular}{lcc}
\hline Parameter/reactor condition & HZP & HFP \\
\hline Fuel temperature, $[\mathrm{K}]$ & 552.833 & 900 \\
Cladding temperature, $[\mathrm{K}]$ & 552.833 & 600 \\
Moderator (coolant) temperature, $[\mathrm{K}]$ & 552.833 & 557 \\
Moderator (coolant) density, $\left[\mathrm{kg} / \mathrm{m}^{3}\right]$ & 753.978 & 460.72 \\
Reactor Power , [MWt] & 3.293 & 3293 \\
Void fraction $(\%)$ & - & 40 \\
\hline
\end{tabular}

Figure 1: Configuration of PB-2 BWR unit cell.

\begin{tabular}{ll} 
& \\
\cline { 2 - 3 } \\
\cline { 2 - 3 }
\end{tabular}

$p$-pitch of the unit cell

\begin{tabular}{lcc}
\hline Parameter/reactor condition & HZP & HFP \\
\hline Fuel temperature, $[\mathrm{K}]$ & 551 & 900 \\
Cladding temperature, $[\mathrm{K}]$ & 551 & 600 \\
Moderator (coolant) temperature, $[\mathrm{K}]$ & 551 & 562 \\
Moderator (coolant) density, $\left[\mathrm{kg} / \mathrm{m}^{3}\right]$ & 766 & 748.4 \\
Reactor power, $[\mathrm{MWt}]$ & 2.772 & 2772 \\
Void fraction $(\%)$ & 551 & 900 \\
\hline
\end{tabular}

FIgUre 2: Configuration of TMI-1 PWR unit cell. 


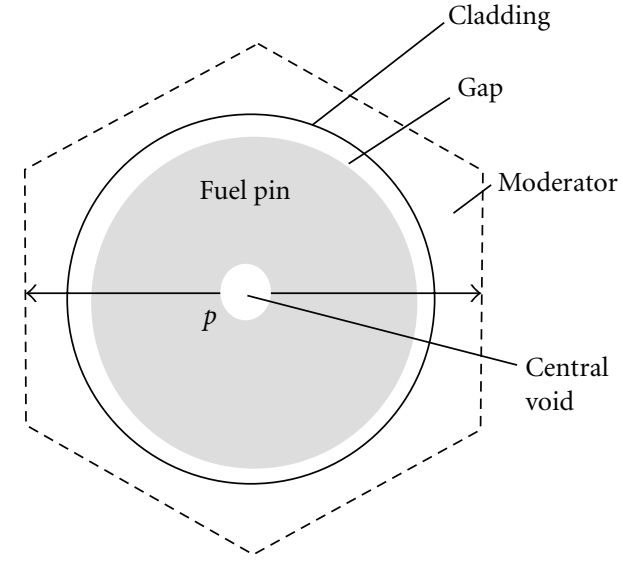

$p$-pitch of the unit cell

\begin{tabular}{lc}
\hline Parameter & Value \\
\hline Unit cell pitch, $[\mathrm{mm}]$ & 12.75 \\
Fuel pellet diameter, $[\mathrm{mm}]$ & 7.56 \\
Fuel pellet material & $\mathrm{UO}_{2}$ \\
Fuel density, $\left[\mathrm{g} / \mathrm{cm}^{3}\right]$ & 10.4 \\
Fuel enrichment, $[\mathrm{w} / \mathrm{o}]$ & 3.3 \\
Central void diameter, $[\mathrm{mm}]$ & 1.4 \\
Central void material & dry air \\
Cladding outside diameter, $[\mathrm{mm}]$ & 9.1 \\
Cladding thickness, $[\mathrm{mm}]$ & 0.69 \\
Cladding material & $\mathrm{Zr}+1 \% \mathrm{Nb}$ \\
Cladding density, $\left[\mathrm{g} / \mathrm{cm}^{3}\right]$ & $\mathrm{n} / \mathrm{a}$ \\
Gap material & $\mathrm{He}$ \\
Moderator material & $\mathrm{H}_{2} \mathrm{O}$ \\
\hline
\end{tabular}

\begin{tabular}{lcc}
\hline Parameter/reactor condition & HZP & HFP \\
\hline Fuel temperature, [K] & 552.15 & 900 \\
Cladding temperature, [K] & 552.15 & 600 \\
Moderator (coolant) temperature, [K] & 552.15 & 560 \\
Moderator (coolant) density, $\left[\mathrm{kg} / \mathrm{m}^{3}\right]$ & 767 & 752.5 \\
Reactor Power , [MWt] & 3.000 & 3000 \\
Void fraction (\%) & 552.15 & 900 \\
\hline
\end{tabular}

Figure 3: Configuration of Kozloduy-6 VVER-1000 unit cell.

TABle 2: Manufacturing tolerances for TMI-1 test case.

\begin{tabular}{lccc}
\hline Parameter & Reference value & TMI-1 & Variation \\
\hline Fuel density & $10.283 \mathrm{~g} / \mathrm{cm}^{3}$ & $\pm 0.17 \mathrm{~g} / \mathrm{cm}^{3}$ & $\pm 0.91 \%\left(= \pm 0.095 \mathrm{~g} / \mathrm{cm}^{3}\right)$ \\
Fuel pellet diameter & $9.391 \mathrm{~mm}$ & $\pm 0.013 \mathrm{~mm}$ & $\pm 0.013 \mathrm{~mm}$ \\
Gap thickness & $0.0955 \mathrm{~mm}$ & $\pm 0.024 \mathrm{~mm}$ & - \\
Clad thickness & $0.673 \mathrm{~mm}$ & $\pm 0.025 \mathrm{~mm}$ & $\pm 0.04 \mathrm{~mm}$ \\
${ }^{235} \mathrm{U}$ concentration & $4.85 \mathrm{w} / \mathrm{o}$ & $\pm 0.00224 \mathrm{w} / \mathrm{o}$ & - \\
\hline
\end{tabular}

$k_{\text {inf }}$ has been converted into reactivity using the following expression:

$$
\rho=\frac{k_{\text {inf }}-1}{k_{\text {inf }}} .
$$

The results from HELIOS and NEWT are compared for TMI-1 pin-cell on Table 4 . The $k_{\text {inf }}$ value calculated by HELIOS is lower than the NEWT one, both for HZP $(-550 \mathrm{pcm})$ and HFP $(-840 \mathrm{pcm})$ conditions. These discrepancies can be due to the different cross-section libraries used by the two codes and the different energy group structures (Table 1).

The difference between HZP and HFP values is of about $1200 \mathrm{pcm}$ in NEWT evaluations and $900 \mathrm{pcm}$ in HELIOS ones. Criticality results for PB-2 and Kozloduy- 6 test cases are provided in Tables 5 and 6.

\subsection{Numerical Discretization Error}

5.2.1. Spatial Discretization Error. One of the user-defined values is a computational grid in which the NEWT ESC solution algorithm is applied. Convergence study has been performed on $k_{\text {inf }}$ value for the grid dimension from $2 \times 2$ (base model) up to $40 \times 40$ per cell. The finer discretization results in lower spatial discretization error, however it requires larger computational effort. Figure 4 shows the reactivity deviation (in $\mathrm{pcm}$ ) from the asymptotic $k_{\text {inf }}$ value (grid $40 \times 40$ ) against the grid dimension for the three test cases.

For all the three test cases increasing the number of computational points the $k_{\text {inf }}$ converges, but the convergence is faster for the Kozloduy- 6 lattice. The $k_{\text {inf }}$ convergence behavior is similar for the TMI-1 and the PB-2 lattice, but it differs from the Kozloduy one. Coarse grid overestimates the $k_{\text {inf }}$ for BWR and PWR and underestimates the $k_{\text {inf }}$ for the VVER. This is most likely because of the different types of lattice: PWR and BWR is a square lattice while the VVER is a hexagonal lattice. 
TABle 3: Manufacturing tolerances for Kozloduy-6 test case.

\begin{tabular}{lccc}
\hline Parameter & Reference value & Lower limit & Upper limit \\
\hline Inner hole diameter & $1.4 \mathrm{~mm}$ & $1.4 \mathrm{~mm}$ & $1.7 \mathrm{~mm}$ \\
Fuel density & $10.4 \mathrm{~g} / \mathrm{cm}^{3}$ & $10.4 \mathrm{~g} / \mathrm{cm}^{3}$ & $10.7 \mathrm{~g} / \mathrm{cm}^{3}$ \\
Fuel pellet diameter & $7.56 \mathrm{~mm}$ & $7.53 \mathrm{~mm}$ & $7.56 \mathrm{~mm}$ \\
Clad inner diameter & $7.72 \mathrm{~mm}$ & $7.72 \mathrm{~mm}$ & $7.78 \mathrm{~mm}$ \\
Clad outer diameter & $9.1 \mathrm{~mm}$ & $9.05 \mathrm{~mm}$ & $9.15 \mathrm{~mm}$ \\
${ }^{235} \mathrm{U}$ concentration & $3.3 \mathrm{w} / \mathrm{o}$ & $3.25 \mathrm{w} / \mathrm{o}$ & $3.35 \mathrm{w} / \mathrm{o}$ \\
\hline
\end{tabular}

TABLE 4: TMI-1 fuel pin-NEWT and HELIOS results for reference model.

\begin{tabular}{lcccc}
\hline & \multicolumn{2}{c}{ HZP } & \multicolumn{2}{c}{ HFP } \\
& NEWT & HELIOS & NEWT & HELIOS \\
\hline$k_{\text {inf }}$ & 1.41481 & 1.42595 & 1.39138 & 1.40788 \\
Reactivity $\rho(\mathrm{pcm})$ & 29319 & 29871 & 28129 & 28971 \\
\hline
\end{tabular}

TABLE 5: PB-2 fuel pin-NEWT results for reference model.

\begin{tabular}{lcc}
\hline & HZP & HFP \\
& NEWT & NEWT \\
\hline$k_{\text {inf }}$ & 1.33869 & 1.21906 \\
Reactivity $\rho(\mathrm{pcm})$ & 25300 & 17932 \\
\hline
\end{tabular}

TABLE 6: Kozloduy-6 fuel pin-NEWT results for reference model.

\begin{tabular}{lcc}
\hline & HZP & HFP \\
& NEWT & NEWT \\
\hline$k_{\text {inf }}$ & 1.34311 & 1.32530 \\
Reactivity $\rho(\mathrm{pcm})$ & 25546 & 24546 \\
\hline
\end{tabular}

It is important to note that the value corresponding to a $2 \times 2$ grid is very far from the converged value, especially for BWR and PWR. In fact, grids lower than $10 \times 10$ have numerical error larger than $100 \mathrm{pcm}$.

For subsequent calculations a $3 \times 3$ computational grid has been chosen for BWR and PWR, taking into account both the accuracy of results and the computational time (that increases with the grid dimension). The reason for such coarse grid is that it is typically used for lattice calculations and recommended by the NEWT manual. $6 \times 6$ grid has been chosen for VVER-1000.

5.2.2. Circle Polygon Approximation Error. Another important NEWT user-defined value is the approximation of a circle with an equilateral polygon with a certain number of sides. Convergence study has been performed on $k_{\text {inf }}$ for the number of polygon sides from 8 to 28 (the default values are 12). Figure 5 shows the reactivity deviation (in $\mathrm{pcm}$ ) from the asymptotic $k_{\text {inf }}$ value (28 sides) against the number of polygon sides for the three test cases.

Nonmonotonic convergence is observed when varying the number of polygon sides, in particular for TMI-1 and PB-2 cases, while for Kozloduy- 6 trend is much more monotonic. A possible reason for such behavior is that the
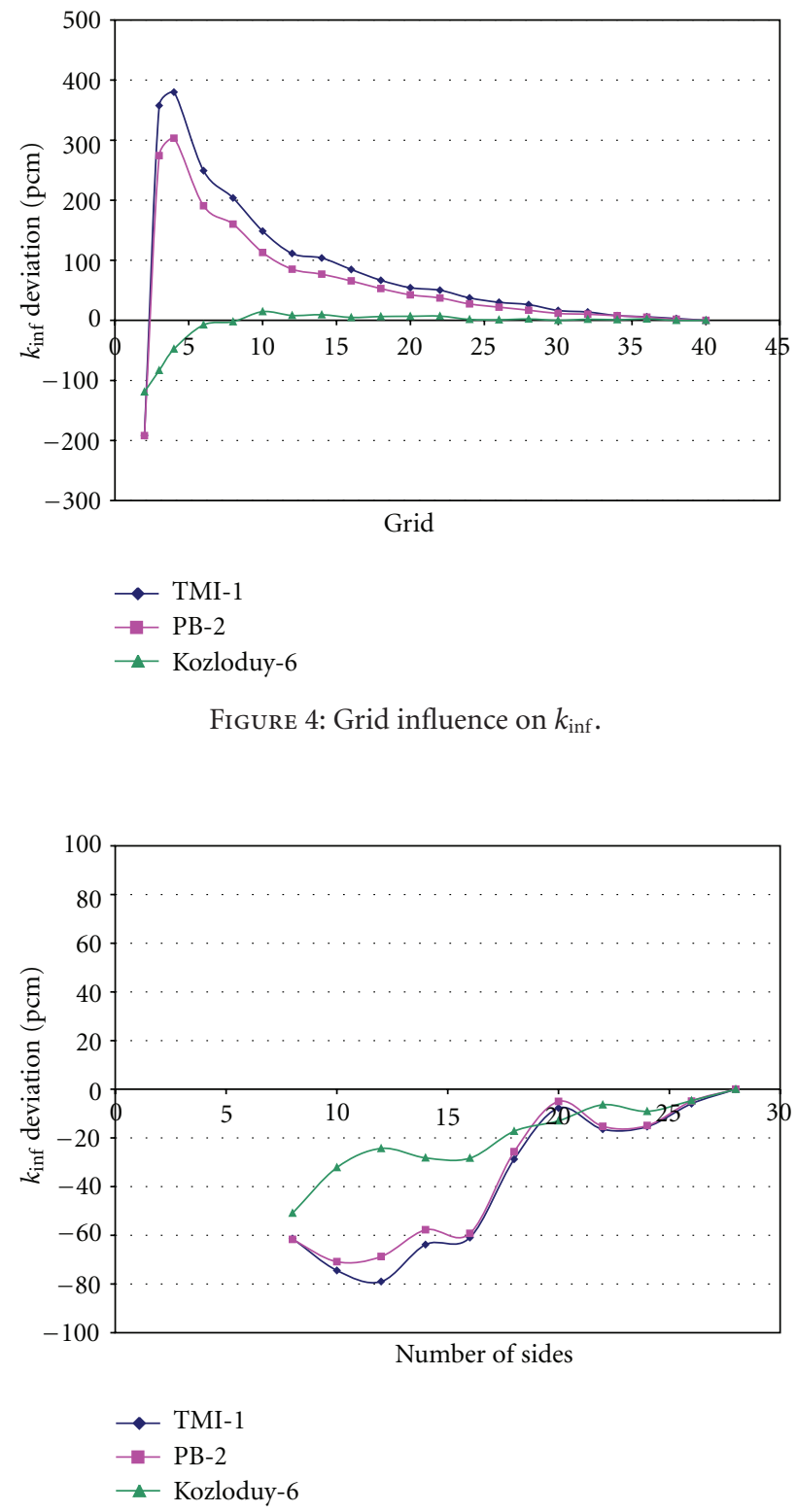

FIGURE 5: Number of polygon sides' influence on $k_{\text {inf }}(3 \times 3$ grid $)$.

use of the $3 \times 3$ grid that is too coarse for a square lattice. Therefore, the same convergence study was performed using the finest grid $(40 \times 40)$, in order to eliminate the possible grid effect. The corresponding result is shown on Figure 6. 
TABLE 7: Manufacturing sensitivities for TMI-1 and PB-2 test cases.

\begin{tabular}{|c|c|c|c|c|c|c|}
\hline \multirow{3}{*}{ Parameter } & \multicolumn{3}{|c|}{ TMI-1 } & \multicolumn{3}{|c|}{ PB-2 } \\
\hline & \multirow[t]{2}{*}{ Variation } & \multicolumn{2}{|c|}{$\Delta \rho(\mathrm{pcm})$} & \multirow[t]{2}{*}{ Variation } & \multicolumn{2}{|c|}{$\Delta \rho(\mathrm{pcm})$} \\
\hline & & $\mathrm{HZP}$ & HFP & & $\mathrm{HZP}$ & HFP \\
\hline Fuel density & $+0.17 \mathrm{~g} / \mathrm{cm}^{3}$ & -97 & -104 & $+0.91 \%$ & -43 & -95 \\
\hline Fuel pellet diameter & $+0.013 \mathrm{~mm}$ & -19 & -20 & $+0.013 \mathrm{~mm}$ & -12 & -23 \\
\hline \multicolumn{7}{|l|}{ Gap thickness } \\
\hline from outside & $+0.024 \mathrm{~mm}$ & -84 & -91 & - & - & - \\
\hline from inside & $+0.024 \mathrm{~mm}$ & 69 & 74 & - & - & - \\
\hline \multicolumn{7}{|l|}{ Clad thickness } \\
\hline from outside & $+0.025 \mathrm{~mm}$ & -104 & -110 & $+0.04 \mathrm{~mm}$ & -129 & -226 \\
\hline from inside & $+0.025 \mathrm{~mm}$ & -16 & -15 & $+0.04 \mathrm{~mm}$ & -23 & -24 \\
\hline${ }^{235} \mathrm{U}$ concentration & $+0.00224 \mathrm{w} / \mathrm{o}$ & 4 & 4 & - & - & - \\
\hline
\end{tabular}

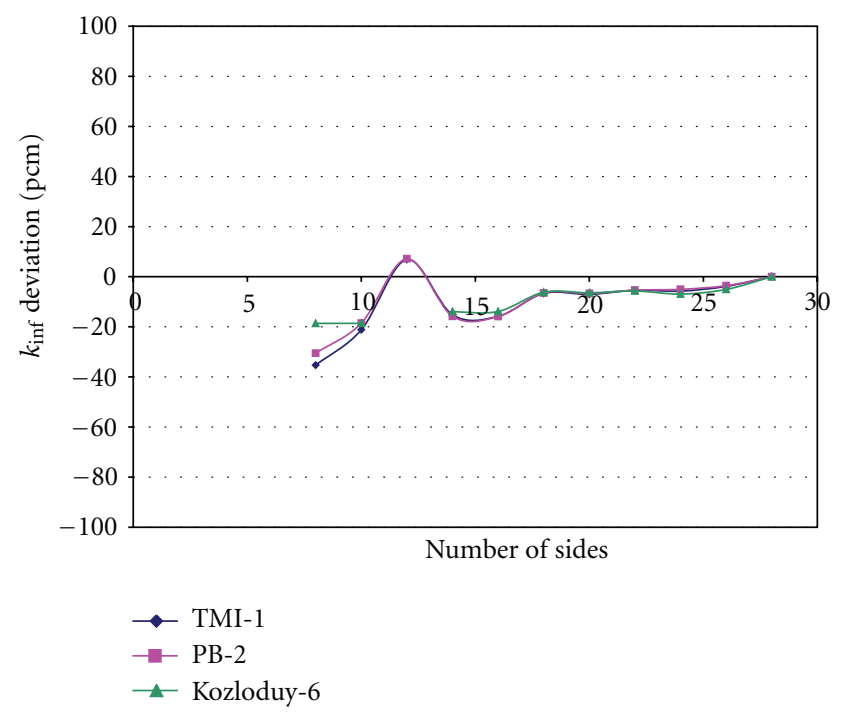

FIgURE 6: Number of polygon sides' influence on $k_{\text {inf }}(40 \times 40$ grid $)$.

With the $40 \times 40$ grid all the test cases have the same asymptotic trend after the first fluctuation. The polygon approximation error is much lower on a finer mesh (compare Figures 5 and 6). It is interesting to note that this fluctuation appears for the default number of polygon sides (12). For the following calculations the default sides' number (12) will be always used.

An important effect is that the spatial discretization error (Figure 4) is opposite to the polygon approximation error (Figure 5). This demonstrates that relatively coarse spatial mesh and coarse polygon approximation can produce good criticality results due to fortunate cancelation of error.

5.3. Manufacturing Tolerance Sensitivities. Sensitivity of lattice-averaged parameters to manufacturing tolerances has been studied for the 3 test cases. For TMI-1 and PB- 2 cases the sensitivities were calculated by increasing the parameters listed in Table 2. For VVER-1000 case the sensitivities were calculated using both the lower and the upper limits of the parameters listed in Table 3 . The $k_{\text {inf }}$ sensitivities were
TABLE 8: Manufacturing sensitivities for Kozloduy-6 test case.

\begin{tabular}{lccc}
\hline & & \multicolumn{2}{c}{$\Delta \rho(\mathrm{pcm})$} \\
Parameter & Variation & \multicolumn{2}{c}{ Kozloduy-6 } \\
& & HZP & HFP \\
\hline Inner hole diameter & & & \\
$\quad$ Upper limit $(0.17 \mathrm{~cm})$ & $+0.03 \mathrm{~cm}$ & & \\
Fuel density & & & \\
$\quad$ Upper limit $\left(10.7 \mathrm{~g} / \mathrm{cm}^{3}\right)$ & $+0.3 \mathrm{~g} / \mathrm{cm}^{3}$ & -151 & -163 \\
Fuel pellet diameter & & & \\
$\quad$ Lower limit $(0.753 \mathrm{~cm})$ & $-0.003 \mathrm{~cm}$ & 50 & 54 \\
Clad inner diameter & & & \\
$\quad$ Upper limit $(0.778 \mathrm{~cm})$ & $+0.006 \mathrm{~cm}$ & 31 & 31 \\
Clad outer diameter & & & \\
$\quad$ Upper limit $(0.915 \mathrm{~cm})$ & $+0.005 \mathrm{~cm}$ & -135 & -144 \\
$\quad$ Lower limit $(0.905 \mathrm{~cm})$ & $-0.005 \mathrm{~cm}$ & 132 & 141 \\
${ }^{235}$ U concentration & & & \\
$\quad$ Upper limit $(3.35 \%)$ & $-0.05 \mathrm{w} / \mathrm{o}$ & 168 & 170 \\
$\quad$ Lower limit $(3.25 \%)$ & $+0.05 \mathrm{w} / \mathrm{o}$ & -173 & -175 \\
\hline
\end{tabular}

TABLE 9: Manufacturing sensitivities on macroscopic cross-sections for TMI-1 fuel pin lattice.

\begin{tabular}{lcccc}
\hline Parameter & Variation & $\begin{array}{c}\Delta \Sigma_{a} \\
\%\end{array}$ & $\begin{array}{c}\Delta \Sigma_{f} \\
\%\end{array}$ & $\begin{array}{c}\Delta \Sigma_{s} \\
\%\end{array}$ \\
\hline Fuel density & $+0.17 \mathrm{~g} / \mathrm{cm}^{3}$ & $1.16 \%$ & $-1.22 \%$ & $-1.16 \%$ \\
Fuel pellet diameter & $+0.013 \mathrm{~mm}$ & $0.22 \%$ & $-0.24 \%$ & $-0.22 \%$ \\
Gap thickness & & & & \\
$\quad$ from outside & $+0.024 \mathrm{~mm}$ & $-0.08 \%$ & $0.07 \%$ & $0.08 \%$ \\
$\quad$ from inside & $+0.024 \mathrm{~mm}$ & $-0.81 \%$ & $0.87 \%$ & $0.81 \%$ \\
Clad thickness & & & & \\
$\quad$ from outside & $+0.025 \mathrm{~mm}$ & $-0.10 \%$ & $0.10 \%$ & $0.10 \%$ \\
$\quad$ from inside & $+0.025 \mathrm{~mm}$ & $-0.02 \%$ & $0.02 \%$ & $0.02 \%$ \\
${ }^{235} \mathrm{U}$ concentration & $+0.00224 \mathrm{w} / \mathrm{o}$ & $0.03 \%$ & $-0.03 \%$ & $-0.03 \%$ \\
\hline
\end{tabular}

calculated for the three cases at HZP and HFP conditions. The macroscopic 2 group cross-sections (absorption, fission, and scattering) sensitivities were calculated only for TMI-1 at HZP conditions. 


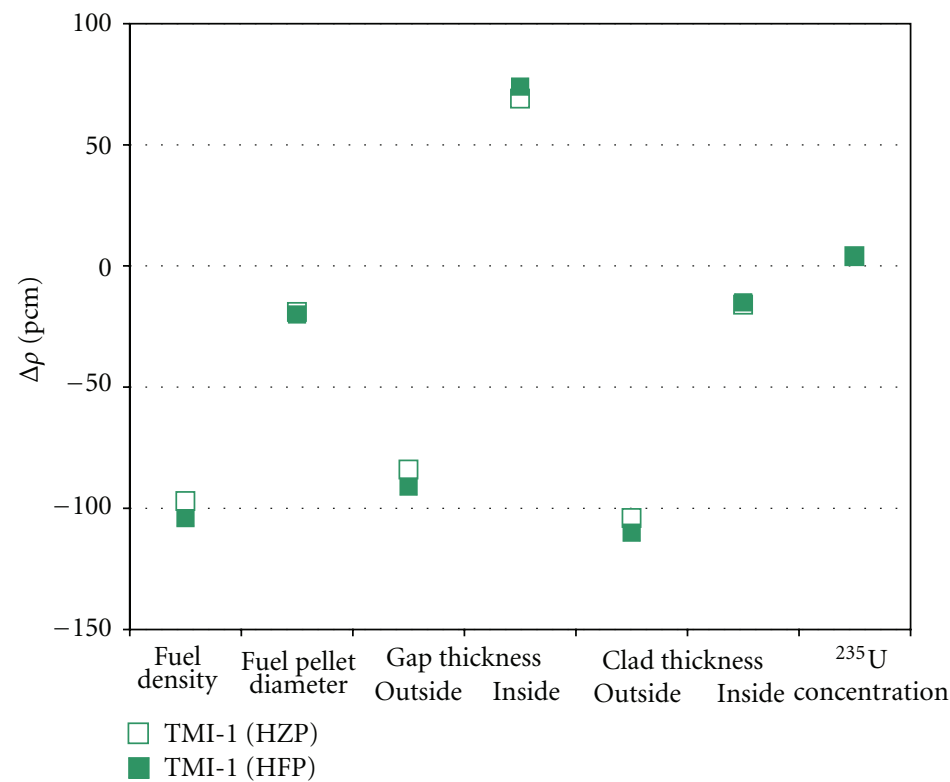

FIgURE 7: Manufacturing sensitivities for TMI-1 test case.

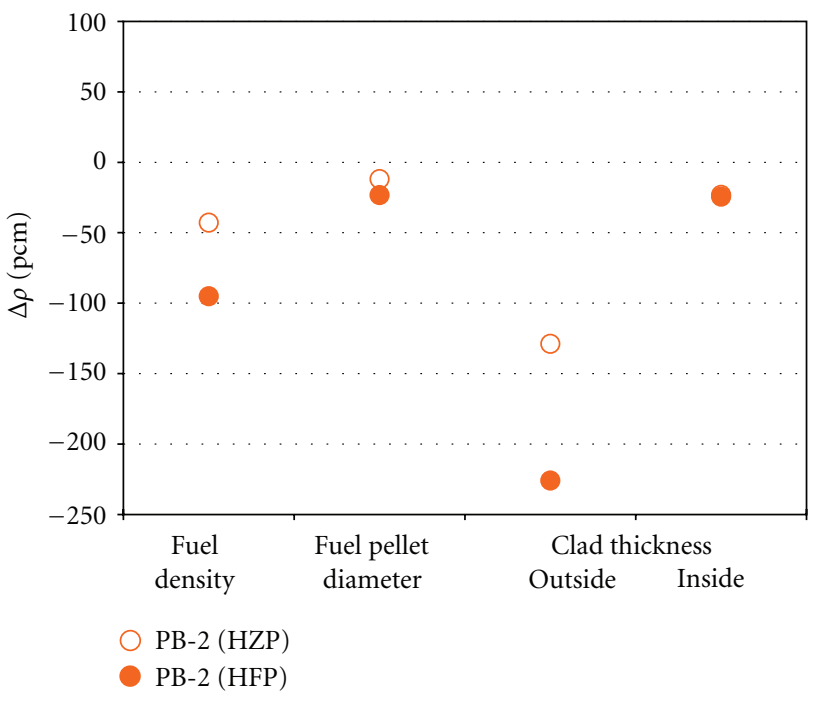

Figure 8: Manufacturing sensitivities for PB-2 test case.

Table 7 shows $k_{\text {inf }}$ sensitivity due to the manufacturing tolerances for TMI-1 and PB-2 test cases at HZP and HFP conditions. The same results are shown graphically on Figures 7 and 8 .

It should be noted that errors in gap and clad thickness can be considered in two ways.

(1) The gap thickness can increase because the fuel pellet diameter decreases or the clad thickness decreases (increase of the internal clad diameter);

(2) The clad thickness can increase because the gap thickness decrease (while outer clad diameter remains the same) or the outer clad diameter increases (while the inner clad diameter remains the same).
Each of the gap and clad thickness variations have been analyzed and are included in Table 7 and Figures 7 and 8.

The largest $k_{\text {inf }}$ sensitivity (absolute values) for both the TMI-1 and PB-2 occur for the manufacturing tolerance of clad thickness (by changing the outer diameter) and fuel density. For all parameters HFP conditions sensitivities are larger (in magnitude) than HZP conditions, especially for the PB-2 test case. The highest $k_{\text {inf }}$ sensitivity (absolute value) due to manufacturing tolerances are $110 \mathrm{pcm}$ for TMI-1 and $226 \mathrm{pcm}$ for PB-2. Further calculations show that $\Delta k$ values change linearly with manufacturing tolerances for all the parameters considered.

Kozloduy- $6 k_{\text {inf }}$ sensitivities due to the manufacturing tolerances are shown on Table 8 and Figure 9. In this case the largest $k_{\text {inf }}$ sensitivity occurs for manufacturing tolerance of fuel density and ${ }^{235} \mathrm{U}$ concentration. These variations have an absolute value of about $150-170 \mathrm{pcm}$.

The same sensitivity calculations have been performed using a $40 \times 40$ computational grid. It was observed that the $k_{\text {inf }}$ sensitivities remain the same for all the test cases. Therefore, the spatial discretization has an effect on $k_{\text {inf }}$ (see Figure 4), but it does not have an effect on $k_{\text {inf }}$ sensitivity due to manufacturing tolerance.

Table 9 contains manufacturing sensitivities on macroscopic 1 group cross-sections $(\Sigma)$ for TMI-1 case in HZP conditions. The manufacturing tolerances have relatively small influence on macroscopic cross-sections. The highest variation is about $1.2 \%$ for the manufacturing tolerance of fuel density.

5.4. Thermal Expansion Effect. The geometric data used so far for our analysis corresponds to the atmospheric temperature, even for HFP calculations. At high temperatures, corresponding to the HFP conditions, both the fuel pellet and the clad undergo thermal expansion. To analyze 


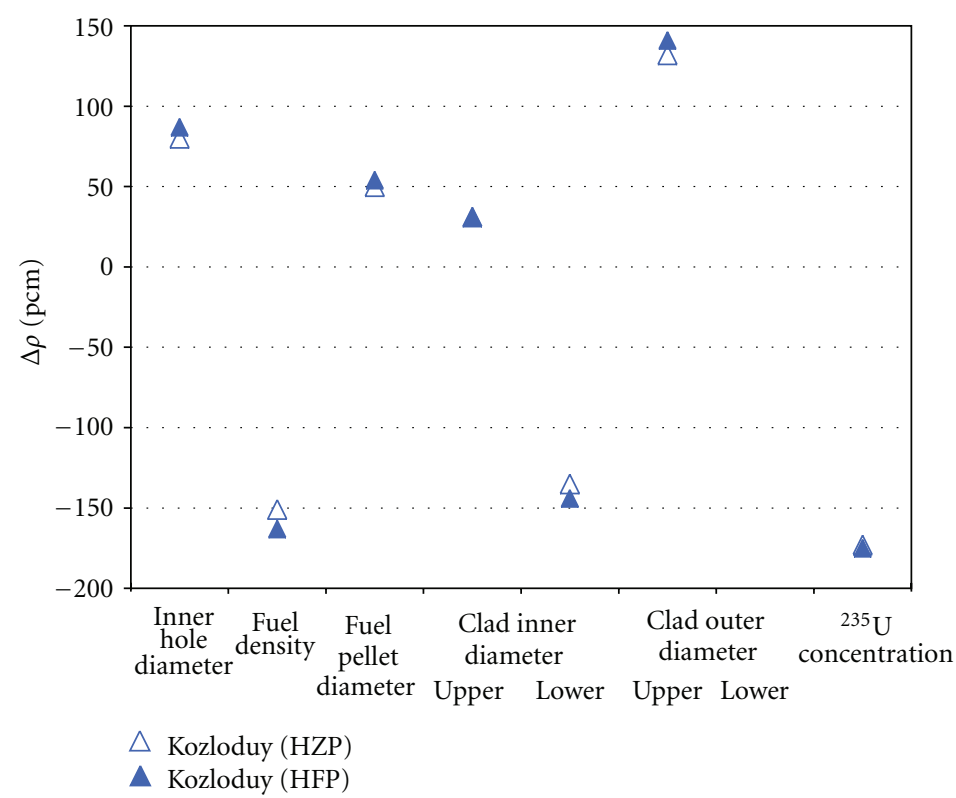

FIGURE 9: Manufacturing sensitivities for Kozloduy-6 test case.

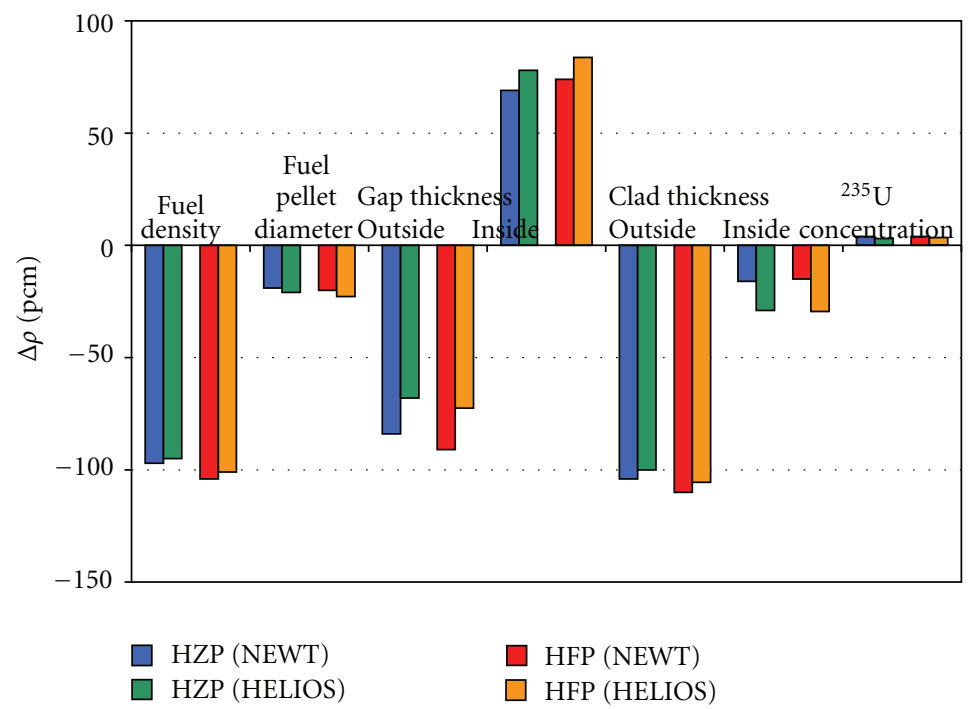

Figure 10: Comparison between HELIOS and NEWT sensitivities for TMI-1.

this effect, we calculated the actual fuel pellet diameter and clad dimensions following the thermal expansion (at HFP temperatures) [4] and recalculated the manufacturing tolerance sensitivities with these new geometric data at HFP conditions. This is to determine if the manufacturing tolerance sensitivities calculated with "cold" dimensions are consistent with the HFP dimensions.

The "hot" pin dimensions have been calculated using a thermal expansion coefficient for the fuel of $17.5 \times 10^{-6} / \mathrm{K}$ and a thermal expansion coefficient for the clad of $6.1 \times$ $10^{-6} / \mathrm{K}$. In order to maintain consistent geometry, we verified that the fuel pellet diameter at HFP temperature plus its manufacturing tolerance remains smaller than the internal clad diameter.
After the calculation of "hot" pin dimension, the manufacturing tolerances sensitivities have been recalculated, at HFP conditions, as before (cf. Section 4.2).

The new results have been compared with the original sensitivities obtained with "cold" dimensions and they are shown in Table 10.

The reactivity sensitivities are the same as those calculated with the "cold" geometry.

5.5. Comparison between NEWT and HELIOS Results for TMI-1. The TMI-1 sensitivities have been recalculated with lattice code HELIOS and compared with the NEWT results. The results are summarized in Table 11 and shown graphically on Figure 10. Both codes predictions are consistent 
TABLE 10: Manufacturing sensitivities for TMI-1 at HFP conditions considering thermal expansion.

\begin{tabular}{lccc}
\hline Parameter & Variation & \multicolumn{2}{c}{$\begin{array}{c}\Delta \rho(\mathrm{pcm}) \\
\text { HFP with thermal } \\
\text { expansion }\end{array}$} \\
\hline Fuel density & $+0.17 \mathrm{~g} / \mathrm{cm}^{3}$ & -104 & -105 \\
$\begin{array}{l}\text { Fuel pellet diameter } \\
\text { Gap thickness }\end{array}$ & $+0.013 \mathrm{~mm}$ & -20 & -20 \\
$\quad$ from outside & $+0.024 \mathrm{~mm}$ & -91 & -92 \\
$\quad$ from inside & $+0.024 \mathrm{~mm}$ & 74 & 74 \\
Clad thickness & & & -111 \\
$\quad \begin{array}{l}\text { from outside } \\
\text { from inside }\end{array}$ & $+0.025 \mathrm{~mm}$ & -110 & -15 \\
${ }^{235} \mathrm{U}$ concentration & $+0.00224 \mathrm{w} / \mathrm{o}$ & 4 & 4 \\
\hline
\end{tabular}

TABle 11: Comparison between HELIOS and NEWT sensitivities for TMI-1.

\begin{tabular}{lccccc}
\hline & & \multicolumn{4}{c}{$\Delta \rho(\mathrm{pcm})$} \\
Parameter & Variation & \multicolumn{2}{c}{ NEWT } & HELIOS \\
& & HZP & HFP & HZP & HFP \\
\hline Fuel density & $+0.17 \mathrm{~g} / \mathrm{cm}^{3}$ & -97 & -104 & -95 & -101 \\
Fuel pellet diameter & $+0.013 \mathrm{~mm}$ & -19 & -20 & -21 & -23 \\
Gap thickness & & & & & \\
$\quad$ from outside & $+0.024 \mathrm{~mm}$ & -84 & -91 & -68 & -73 \\
$\quad$ from inside & $+0.024 \mathrm{~mm}$ & 69 & 74 & 78 & 84 \\
Clad thickness & & & & & \\
$\quad$ from outside & $+0.025 \mathrm{~mm}$ & -104 & -110 & -100 & -106 \\
$\quad$ from inside & $+0.025 \mathrm{~mm}$ & -16 & -15 & -29 & -30 \\
${ }^{235} \mathrm{U}$ concentration & $+0.00224 \mathrm{w} / \mathrm{o}$ & 4 & 4 & 3 & 4 \\
\hline
\end{tabular}

with each other. The highest discrepancy between the two is only $18 \mathrm{pcm}$. Therefore, even though the absolute value of reactivity differs by $100 \mathrm{~s} \mathrm{pcm}$ (see Table 4 ), the sensitivities are practically the same.

\section{Conclusions}

This work has been carried out in the framework of UAM Exercise I-1 "Cell Physics." Three test cases (TMI-1, PB-2, and Kozloduy-6) have been analyzed with the deterministic code NEWT. In addition, the TMI-1 fuel pin has also been modeled with the HELIOS code in order to compare the results of the two codes.

The infinite multiplication factor has been calculated for each of the lattice configuration. A significant discrepancy was found in the multiplication factor between NEWT and HELIOS for the TMI-1 case. The difference was about $600 \mathrm{pcm}$ at HZP conditions and about $900 \mathrm{pcm}$ at HFP conditions.

Sensitivity calculations have been performed in order to study the influence of numerical approximations and manufacturing tolerances on $k_{\text {inf }}$. The spatial discretization error was quantified for $k_{\mathrm{inf}}$ in the NEWT code and the manufacturing sensitivities have been performed using data from UAM specifications for Phase I.

The following important conclusions related to the NEWT numerical approximation can be highlighted.

(i) The spatial discretization error for $k_{\text {inf }}$ shows an asymptotic convergence. The difference between the coarsest and the finest grid is about $300-400 \mathrm{pcm}$ for TMI- 1 and PB-2 test cases, and about $100 \mathrm{pcm}$ for Kozloduy-6 test case.

(ii) The spatial discretization error is very large with the default discretization $(2 \times 2$ or $3 \times 3$ grid $)$, it is larger than any of the manufacturing sensitivities.

(iii) The equilateral polygon approximation of a circle has relatively small influence on $k_{\text {inf }}$. Calculations performed with a coarse grid $(3 \times 3)$ show a fluctuating trend that becomes asymptotic on a finer grid $(40 \times 40)$.

(iv) The spatial discretization error and circle polygon approximation error are in opposite direction, causing a fortunate cancelation of error.

The following important conclusions related to the manufacturing sensitivities can be highlighted.

(i) Sensitivities change linearly with manufacturing tolerances for all the parameters considered.

(ii) HFP sensitivities are larger than HZP ones, especially for PB-2 case.

(iii) The manufacturing tolerance that has the largest influence on the $k_{\text {inf }}$ is the outer clad diameter for TMI- 1 and PB-2 test case and ${ }^{235} \mathrm{U}$ enrichment for Kozloduy- 6 test case. The second most important parameter for all test cases is the fuel density.

(iv) The influence of manufacturing tolerances on two group macroscopic cross-sections has been analyzed and maximum variation is about $1.2 \%$.

(v) Manufacturing tolerances sensitivities with "cold" and "hot" dimensions are the same.

(vi) The spatial discretization has a significant effect on $k_{\text {inf }}$, but it does not have appreciable effect on manufacturing tolerance sensitivities.

(vii) $k_{\text {inf }}$ value is significantly different for HELIOS and NEWT, but the manufacturing tolerance sensitivities are almost the same.

The propagation of manufacturing tolerances for reactivity and few group nodal homogenized data at the fuel assembly level will be performed in the future.

\section{References}

[1] M. D. Dehart, "NEWT: a new transport algorithm for twodimensional discrete ordinates analysis in non-orthogonal geometries," ORNL/TM-2005/39, 2009.

[2] Studsvik-Scandpower, User Manual HELIOS, 2009. 
[3] K. Ivanov, M. Avramova, I. Kodeli, and E. Sartori, "Benchmark for uncertainty analysis in modeling (UAM) for design, operation and safety analysis of LWRs_-volume I: specification and support data for the neutronics cases (phase I)," NEA/NSC/DOC(2012), 2012.

[4] F. A. Halden, H. C. Wohlers, and R. H. Reinhart, "Thermal expansion of uranium dioxide," Final Report SRI/TDI-5722, 1959. 

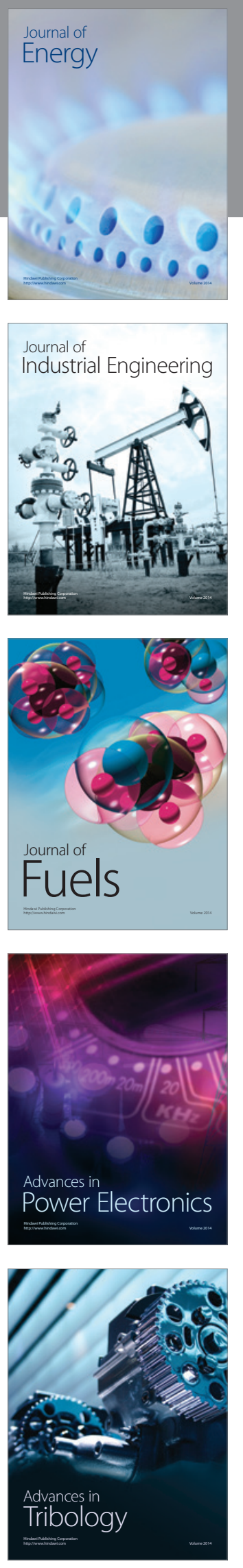
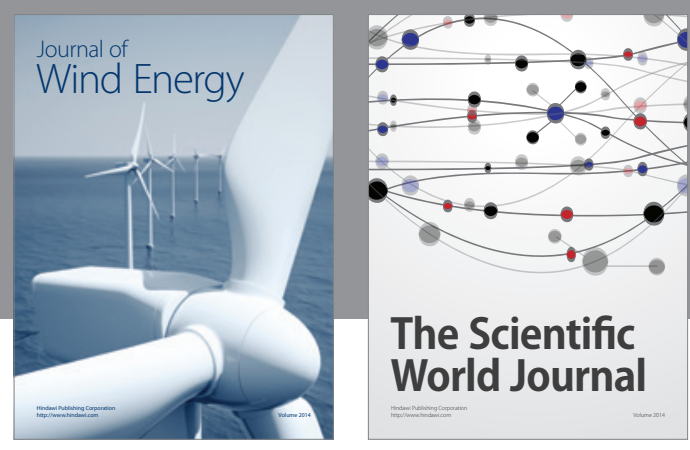

The Scientific World Journal

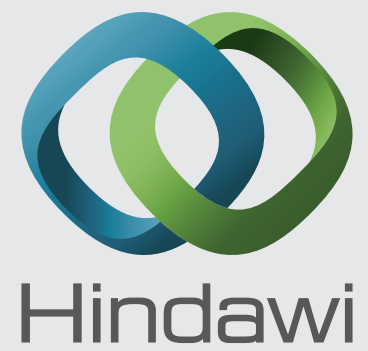

Submit your manuscripts at http://www.hindawi.com
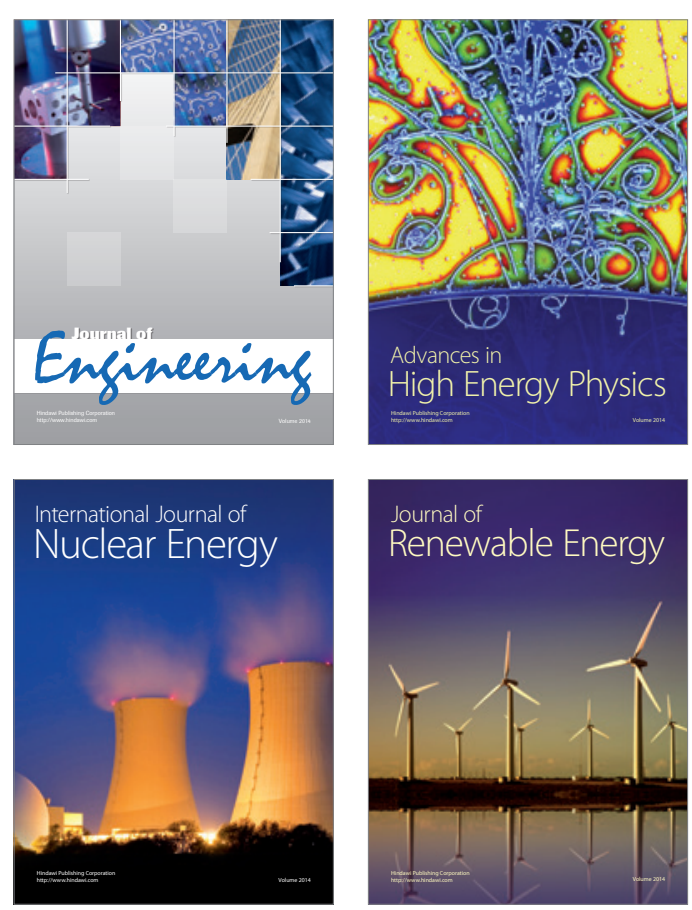

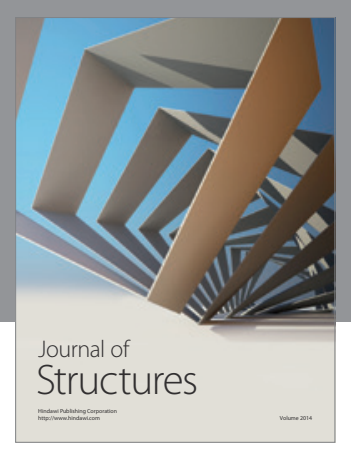

Rotating
Mechinery
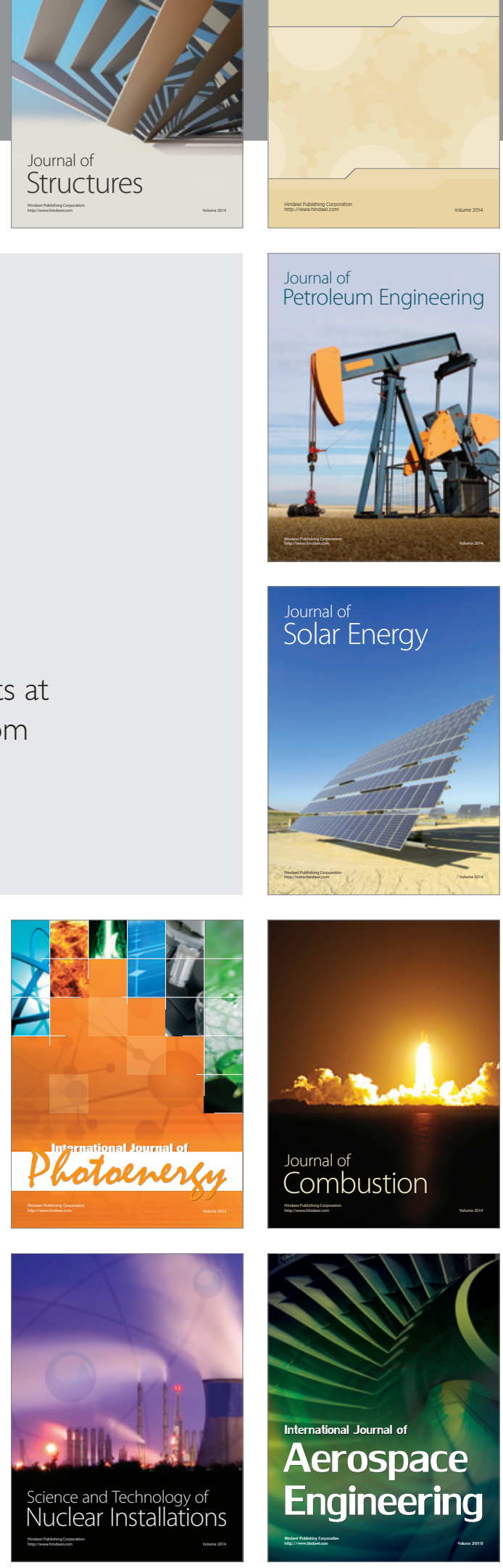